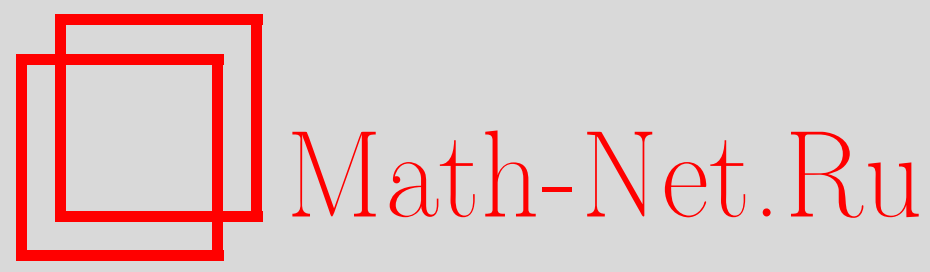

О. М. Пенкин, Е. М. Богатов, О слабой разрешимости задачи Дирихле на стратифицированных множествах, Матем. заметки, 2000, том 68, выпуск 6, 874-886

DOI: https://doi.org/10.4213/mzm1011

Использование Общероссийского математического портала Math-Net.Ru подразумевает, что вы прочитали и согласны с пользовательским соглашением http://www . mathnet.ru/rus/agreement

Параметры загрузки:

IP: 54.172 .240 .79

26 апреля 2023 г., 14:11:41

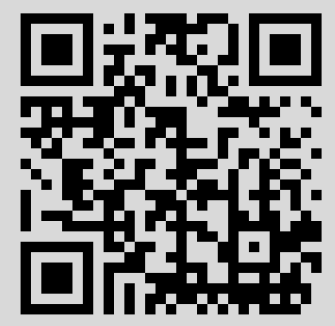




\title{
О СЛАБОЙ РАЗРЕШИМОСТИ ЗАДАЧИ ДИРИХЛЕ НА СТРАТИФИЦИРОВАННЫХ МНОЖЕСТВАХ
}

\author{
О. М. Пенкин, Е. М. Богатов
}

\begin{abstract}
На множествах, составленных из многообразий различных размерностей, правильно примыкающих друг к другу (стратифицированных множествах), ставится задача Дирихле для оператора, аналогичного оператору Лапласа-Бельтрами. Специальный понятийный аппарат позволяет доказать аналоги интегральных тождеств Грина и неравенства Пуанкаре для пространств соболевского типа. Слабая разрешимость задачи Дирихле доказывается на основе теоремы Рисса о представлении. Аналогичным образом устанавливается слабая разрешимость задачи Дирихле для аналога бигармонического оператора на стратифицированных множествах.
\end{abstract}

Библиография: 12 названий.

Изучение статики и динамики механических систем, составленных из элементов разных размерностей, приводит к необходимости рассматривать весьма сложные наборы дифференциальных соотношений, каждое из которых описьвает поведение отдельного элемента. В числе первых работ (близких по тематике к данной) отметим [1]-[3]. Обычно такие наборы трактуются как одно операторное уравнение, определенное на произведении пространств. Детально такой подход описан, например, в [4] (в контексте струнно-мембранных систем) и в [5] (в контексте гидродинамики).

В данной работе мы придерживаемся иной методики. Она основана на интерпретации всей совокупности соотношений в виде единого дифференциального оператора, которьй в случае задачи о деформациях струнно-мембранных систем описывается точным аналогом оператора Лапласа в дивергентной форме (дивергенция понимается нами как плотность потока векторного поля по специальной мере). Следует отметить, что идея рассматривать дивергенцию и пространства Соболева по мере не нова, и представляется странньм, что к рассматриваемому классу задач она стала применяться совсем недавно. Для периодических задач такой подход был применен в [6] в контексте метода усреднения.

В несколько формальном виде данная методика представлена в работах [7], [8]. В более поздних работах [9], [10] она изложена уже в “почти классических" терминах.

Данная работа посвящена доказательству слабой разрешимости эллиптических краевых задач второго и четвертого порядка на стратифицированных множествах (последний термин формализует упомянутые вьше системы составного типа). Для уравнений второго порядка результаты были анонсированы в [11]. 
1. Модельный пример. Прежде чем перейти к строгим определениям, разберем пример задачи, приводящей к эллиптическим уравнениям на стратифицированных множествах. Этот пример будет использоваться нами и далее для иллюстрации вводимых понятий. Рассмотрим на плоскости натянутую механическую систему $\Omega$, изображенную на рис. 1.

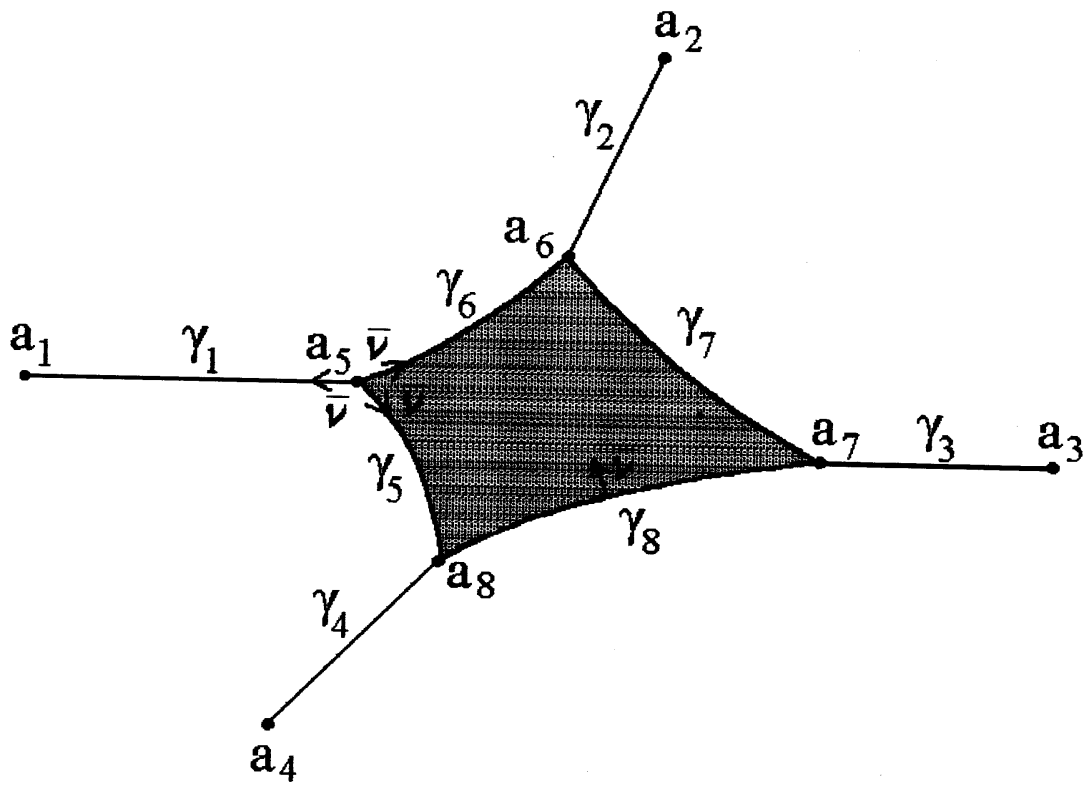

Рис. 1

Здесь $\gamma_{i}$ - струны, на $\gamma_{5}, \ldots, \gamma_{8}$ натянута мембрана $Q, a_{i}(i=\overline{1,4})$ - точки закрепления $\Omega$. Деформации этой системы под воздействием малой поперечной силы $f$ на отрез$\operatorname{kax} \gamma_{1}, \ldots, \gamma_{4}$ описьваются уравнениями

$$
-u^{\prime \prime}=f
$$

На участках $\gamma_{5}, \ldots, \gamma_{8}$ уравнения деформаций вьглядят так:

$$
-u_{\tau}^{\prime \prime}-u_{\nu}^{\prime}=f
$$

где $\nu$ - внутренняя по отношению к четырехугольнику $Q$ нормаль, $\tau$ - направление “вдоль струны". Во внутренних точках $Q$ деформация описывается уравнением Пуассона

$$
-\Delta u=f
$$

где через $\Delta$, как обычно, обозначен оператор Лапласа. Наконец, в местах стыковки струн - точках $a_{i}(i=\overline{5,8})$ - мы имеем уравнения

$$
-\sum \frac{\partial u}{\partial \nu}=f
$$


В последнем равенстве суммирование происходит по примыкающим струнам; направление нормалей указано на рисунке для точки $a_{5}$. Условия закрепления приводят к равенствам $u\left(a_{i}\right)=0(i=\overline{1,4})$, являющимися аналогами условий Дирихле.

Как видим, получается довольно разнородный набор уравнений, с которым не очень удобно работать. Однако за этим набором “стоит" единое эллиптическое уравнение дивергентного типа. Ближайшие пункты посвящены обсуждению этого в самой общей ситуации.

2. Основные обозначения. Здесь мы дадим несколько более точное по сравнению c [9]-[11] определение стратифицированного множества.

Стратификация на множестве $\Omega \subset \mathbb{R}^{n}$ - это набор замкнутых множеств

$$
\Omega^{k_{0}} \subset \Omega^{k_{1}} \subset \cdots \subset \Omega^{k_{p}}=\Omega, \quad 0 \leqslant k_{0}<k_{1}<\cdots<k_{p},
$$

такой, что выполняются следующие условия:

а) множество $\Omega^{k_{i}} \backslash \Omega^{k_{i-1}}$ - гладкое подмногообразие в $\mathbb{R}^{n}$ размерности $k_{i}$; его связные компоненты назьваются стратами размерности $k_{i}$;

б) граница $\partial \sigma_{k i}=\bar{\sigma}_{k i} \backslash \sigma_{k i}$ любого страта ненулевой размерности является объединением стратов менњшей (чем $k$ ) размерности;

в) если страт $\sigma_{k i}$ примькает к $\sigma_{k+1, j}$ и $x \in \sigma_{k i}$, а $y \in \sigma_{k+1, j}$ приближается к $x$ по некоторой непрерывной кривой, расположенной в $\sigma_{k+1, j}$, то касательная плоскость $T_{y}\left(\sigma_{k+1, j}\right)$ (обычная $(k+1)$-мерная плоскость в $\mathbb{R}^{n}$, касающаяся $\sigma_{k+1, j}$ в точке $y$ ) имеет предельное положение $\lim _{y \rightarrow x} T_{y}\left(\sigma_{k+1, j}\right)$, содержащее $T_{x}\left(\sigma_{k i}\right)$.

В нашем примере стратификация такова: $\Omega^{0} \subset \Omega^{1} \subset \Omega^{2}=\Omega$, где $\Omega^{0}$ - множество точек $\left\{a_{i}\right\}_{i=1}^{8}, \Omega^{1}$ получается из $\Omega^{0}$ добавлением $\gamma_{i}(i=\overline{1,8})$, а $\Omega^{2}$ - добавлением внутренности четырехугольника $Q$ к $\Omega^{1}$.

В дальнейшем запись вида $\sigma_{k i} \prec \sigma_{m j}$ означает примыкание страта $\sigma_{k i}$ к $\sigma_{m j}$, т.е. то обстоятельство, что $\sigma_{k i} \subset \bar{\sigma}_{m j}$ и $k<m$. Далее предполагается, что в $\Omega$ конечное число стратов и все они имеют компактные замыкания. Множество $\Omega$ считается связным. Вместе с $\Omega$ мы фиксируем связное открытое (в топологии, индуцированной на $\Omega$ из $\mathbb{R}^{n}$ ) подмножество $\Omega_{0} \subset \Omega$, составленное из стратов и такое, что $\bar{\Omega}_{0}=\Omega$. Положим $\partial \Omega_{0}=\Omega \backslash \Omega_{0}$. Для нашего примера возможно в качестве $\partial \Omega_{0}$ взять множество $\left\{a_{1}, a_{2}, a_{3}, a_{4}\right\}$ или любое его подмножество.

На множестве $\Omega_{0}$ далее будут рассматриваться уравнения, а на $\partial \Omega_{0}$ будут задаваться условия Дирихле.

Пусть $\sigma_{k i} \prec \sigma_{k+1, j}$ и $x \in \sigma_{k i}$. Если $\sigma_{k i}$ примыкает более чем к двум $(k+1)$-мерным клеткам, то ни в какой окрестности $x$ нельзя задать даже локальные координаты. Поэтому координаты мы будем задавать по отдельности для каждой пары $\sigma_{k i}, \sigma_{k+1, j} \succ \sigma_{k i}$, задавая сначала координаты $y^{1}, \ldots, y^{k}$ на $\sigma_{k i}$, а затем дополняя их координатой $y^{k+1}$, своей для каждого $\sigma_{k+1, j}$. Эти координаты мы будем называть правильными, если $y^{k+1} \equiv 0$ на $\sigma_{k i}$ и $y^{k+1}>0$ на $\sigma_{k+1, j}$.

2.1. Мера и интеграл на $\Omega_{0}$. Если сужение функции $f: \Omega \rightarrow \mathbb{R}$ на каждый страт является равномерно непрерьвной функцией (не являясь, вообще говоря, непрерьвной в целом на $\Omega$ ), то будем называть ее постратно непрерывной. Множество таких функций обозначим $\bar{C}_{\sigma}\left(\Omega_{0}\right)$. Для функций из $\bar{C}_{\sigma}\left(\Omega_{0}\right)$ определим интеграл по формуле

$$
\int_{\Omega_{0}} f d \mu=\sum_{\sigma_{k i} \subset \Omega_{0}} \int_{\sigma_{k i}} f d \mu
$$


где в правой части стоят обычные интегралы Римана по стандартной мере на каждом страте, т.е. на $k$-мерном страте $\sigma_{k i}$ мера - это $k$-мерньй объем. При $k=0$ под интегралом от $f$ по $\sigma_{k 0}$ мы понимаем $f(x)$, где $x$ - единственная точка страта $\sigma_{k 0}\left(\sigma_{k 0}=\{x\}\right)$. Как подмножество $\mathbb{R}^{n}$ страт $\sigma_{k i}$ наследует его метрику, становясь, тем самым, римановым многообразием. Зафиксировав в $\sigma_{k i}$ локальные координаты $y^{1}, \ldots, y^{k}$ и обозначив через $g_{i j}$ компоненты римановой метрики в этих координатах, имеем (для малых параллелепипедов $\omega$ со сторонами $\left.d y^{1}, \ldots, d y^{k}\right)$

$$
\mu(\omega)=\sqrt{g} d y^{1} \cdots d y^{k}
$$

где $g$ - определитель матрищы $\left(g_{i j}\right)$. Функции из $\bar{C}_{\sigma}\left(\Omega_{0}\right)$ обязательно ограничены на $\Omega_{0}$, что следует из компактности замыканий стратов и конечности их числа, а потому интеграл (1) существует для любой $f \in \bar{C}_{\sigma}\left(\Omega_{0}\right)$. Формулой

$$
\langle f, g\rangle_{\mu}=\int_{\Omega_{0}} f g d \mu
$$

задается скалярное произведение на $\bar{C}_{\sigma}\left(\Omega_{0}\right)$. Определяемая им норма обозначается через $\|\cdot\|_{\mu}$. Теперь мы можем ввести новое пространство $L_{\mu}^{2}\left(\Omega_{0}\right)$ - пополнение $\bar{C}_{\sigma}\left(\Omega_{0}\right)$ по норме $\|\cdot\|_{\mu}$.

Определение интеграла формулой (1) вьплядит несколько необычно. Тем не менее, оказьвается, что он является интегралом Лебега-Стилтьеса по мере $\mu$ на $\Omega_{0}$, определяемой так: множество $G \subset \Omega_{0}$ назьвается измеримым, если измеримы по Лебегу все пересечения $G \cap \sigma_{k i}\left(\sigma_{k i} \subset \Omega_{0}\right)$. При этом мера $\mu(G)$ полагается равной сумме мер Лебега $\mu\left(G \cap \sigma_{k i}\right)$, т.е. с учетом (2)

$$
\mu(\omega)=\sum \int_{\sigma_{k i} \cap G} \sqrt{g} d y^{1} \cdots d y^{k} .
$$

Такую меру естественно назьвать мерой Лебега-Cтилтьеса (сужение ее на 0-мерные страты - это сосредоточенная мера единичной массы).

2.2. Дивергенция и оператор Лапласа-Бельтрамина $\Omega_{0}$. Здесь нам потребуется еще пространство $C_{\sigma}^{m}\left(\Omega_{0}\right)$ - множество функций, имеющих непрерьвные производные $m$-го порядка по локальным координатам в каждом $\sigma_{k i}$, допускаюшие продолжение по непрерьвности в точки стратов $\sigma_{k-1, j} \not \subset \partial \Omega_{0}$, примыкающих к $\sigma_{k i}$. Мы допускаем, тем самым, "порчу" функций из $C_{\sigma}^{m}\left(\Omega_{0}\right)$ на границе, приспосабливая это пространство к рассмотрению задачи Дирихле.

Пусть $\vec{F}$ - векторное поле на $\Omega_{0}$; включения типа $\vec{F} \in C_{\sigma}^{m}\left(\Omega_{0}\right)$ имеют для нас покоординатньй смысл. Векторное поле $\vec{F}$ будем называть касательным $\kappa \Omega_{0}$, если для любого $x \in \Omega_{0}$ вектор $\vec{F}(x)$ касается страта $\sigma_{k i}$, содержащего $x$, в этой точке, т.е. $\vec{F}(x) \in T_{x} \sigma_{k i}$ (в нульмерных стратах $\vec{F}$ всегда считается нулевым). В дальнейшем по повторяющимся греческим индексам предполагается суммирование, суммирование по иным индексам всегда будет указьваться явно.

Далее запись $\left.f\right|_{k i}$ означает сужение функции $f$ на $\sigma_{k i}$, а для $f \in C_{\sigma}\left(\Omega_{0}\right)$ запись $\left.f\right|_{k i}$ означает продолжение по непрерьвности функции $\left.f\right|_{k i}$ на замькание $\sigma_{k i}$. Для непрерьвных $f$ функция $\left.f\right|_{\overline{k i}}$ совпадает с сужением $f$ на $\bar{\sigma}_{k i}$. 
Пусть $\vec{F} \in C_{\sigma}^{1}\left(\Omega_{0}\right)$ - касательное векторное поле на $\Omega_{0}$ и $x \in \sigma_{k-1, i}$. Дивергенция $\vec{F}$ в точке $x$ определяется формулой

$$
(\nabla \vec{F})(x)=\frac{1}{\sqrt{g}} \frac{\partial}{\partial y^{\alpha}}\left(\sqrt{g} g^{\alpha \beta} F_{\beta}\right)(x)+\left.\sum_{\sigma_{k j} \succ \sigma_{k-1, i}} \frac{1}{\sqrt{g^{k k}}} g^{k \gamma} F_{\gamma}\right|_{\overline{k j}}(x) .
$$

Здесь $g^{\alpha \beta}(\alpha, \beta \leqslant k-1)$ - контравариантные компоненты римановой метрики. Индекс $\gamma$ меняется от 1 до $k$. Сумма в (5) состоит из двух частей, первая из которых - классическая $(k-1)$-мерная дивергенция поля $\vec{F}$ на $\sigma_{k-1, i} ;$ будем обозначать ее $\nabla_{k-1} \vec{F}$. Каждое слагаемое второй суммы является проекцией $\left.\vec{F}\right|_{k j}(x)$ на направление внутренней по отношению к $\sigma_{k j}$ нормали к $\sigma_{k-1, i}$ в точке $x$. Нормаль к $(k-1)$-мерному страту, направленная в $k$-мерньй страт, выражается через риманову метрику следующим образом:

$$
\vec{\nu}=\frac{g^{k \alpha}}{\sqrt{g^{k k}}} \vec{r}_{y^{\alpha}}, \quad \alpha \leqslant k .
$$

Следовательно, (5) можно переписать в виде

$$
(\nabla \vec{F})(x)=\left(\nabla_{k-1} \vec{F}\right)(x)+\left.\sum_{\sigma_{k j} \succ \sigma_{k-1, i}}(\vec{F} \cdot \vec{\nu})\right|_{k j}(x) .
$$

Если примыкание $\sigma_{k-1, i}$ к $\sigma_{k j}$ кратное, то страт $\sigma_{k j}$ участвует в сумме $(5)$ дважды. Отметим, что дивергенция в нульмерном страте совсем не содержит дифференцирований; естественно считать $\nabla_{0} \vec{F}=0$, поэтому $\nabla \vec{F}$ сводится в данном случае ко второму слагаемому в (6).

Взяв в качестве $F_{\alpha}$ компоненты градиента функции $u \in C_{\sigma}^{2}\left(\Omega_{0}\right)$ (в силу соответствующего соглашения об $\vec{F}$ в нульмерных стратах нульмерный градиент считается нулевым), получаем аналог оператора Лапласа-Бельтрами на $\Omega_{0}$; в точке $x \in \sigma_{k-1, i}$ он имеет вид

$$
(\Delta u)(x)=\left(\Delta_{k-1} u\right)(x)+(\nabla u)_{\vec{\nu}}(x),
$$

где $\Delta_{k-1}-$ классический $(k-1)$-мерньй оператор Лапласа-Бельтрами

$$
\left(\Delta_{k-1} u\right)(x)=\frac{1}{\sqrt{g}} \frac{\partial}{\partial y^{\alpha}}\left(\sqrt{g} g^{\alpha \beta} \frac{\partial u}{\partial y^{\beta}}\right)(x),
$$

a

$$
(\nabla u)_{\vec{\nu}}(x)=\left.\sum_{\sigma_{k j} \succ \sigma_{k-1, i}}(\nabla u \cdot \vec{\nu})\right|_{\overline{k j}}(x)=\sum_{\sigma_{k j} \succ \sigma_{k-1, i}}\left(\left.\frac{\partial u}{\partial \nu}\right|_{\overline{k j}}\right)(x) .
$$

Если $x \in \sigma_{1 i}=\gamma_{i}(i=\overline{5,8})$ на рис. 1 , то $\left(\Delta_{k-1} u\right)(x)=u_{\tau}^{\prime \prime}(x)$, a $(\nabla u)_{\vec{\nu}}(x)=u_{\nu}^{\prime}(x)$. Тем самым, уравнение (II) имеет вид $-\Delta u=f$. Подобным образом можно убедиться, что и уравнения (I), (IV) записываются в таком же виде. Лапласиан в уравнении (III), очевидно, совпадает с "новым" лапласианом.

Наряду с $\Delta$ мы будем рассматривать оператор $\Delta_{p}=\nabla(p \nabla)$ при $p \in C_{\sigma}^{1}\left(\Omega_{0}\right)$, т.е.

$$
\left(\Delta_{p} u\right)(x)=\nabla_{k-1}(p \nabla u)(x)+(p \nabla u)_{\vec{\nu}}(x)
$$


где

$$
(p \nabla u)_{\vec{\nu}}(x)=\sum_{\sigma_{k j} \succ \sigma_{k-1, i}}\left(\left.p \frac{\partial u}{\partial \nu}\right|_{\overline{k j}}\right)(x) .
$$

Заметим, что значок $\nabla$ теперь несет двойную нагрузку. Для векторных полей - это дивергенция, для скалярнозначных функций (скалярных полей) - это градиент.

Основной интерес для нас представляет оператор $L_{q} u=\Delta_{p} u-q u$, где $q \in \bar{C}_{\sigma}\left(\Omega_{0}\right)$, a $p$ - строго положительная на $\Omega_{0}$ функция.

3. Вспомогательные аналитические приемы. Через $\bar{C}_{\sigma}^{m}\left(\Omega_{0}\right)$ будем обозначать множество функций из $C_{\sigma}^{m}\left(\Omega_{0}\right)$, производные которых допускают продолжение по непрерьвности и в точки из $\partial \Omega_{0}$.

Всюду далее замыкания стратов считаются ориентируемьми многообразиями, допускающими введение глобальных координат.

Нам потребуются следующие факты.

Теорема 1 (формула Остроградского-Гаусса). Пусть $\vec{F} \in \bar{C}_{\sigma}^{1}\left(\Omega_{0}\right)$. Тогда

$$
\int_{\Omega_{0}} \nabla \vec{F} d \mu=-\int_{\partial \Omega_{0}} \vec{F}_{\vec{\nu}} d \mu, \quad \partial \Omega_{0} \neq \varnothing
$$

әде $\vec{F}_{\vec{\nu}}$ на $ә \Omega_{0}$ определяется следуюшим образом:

$$
\vec{F}_{\vec{\nu}}(x)=\left.\sum(\vec{F} \cdot \vec{\nu})\right|_{k j}(x), \quad x \in \sigma_{k-1, i},
$$

суммирование проводится по всем $j$ таким, что $\sigma_{k j} \succ \sigma_{k-1, i} u \sigma_{k j} \not \subset \partial \Omega_{0}$.

Обозначим через $C\left(\Omega_{0}\right)$ множество непрерывных на $\Omega_{0}$ функций и положим $C^{m}\left(\Omega_{0}\right)$ $=C_{\sigma}^{m}\left(\Omega_{0}\right) \cap C\left(\Omega_{0}\right)$.

Из (7) вытекает аналог первой формулы Грина.

Теорема 2. Пусть $u \in \bar{C}_{\sigma}^{2}\left(\Omega_{0}\right), v \in \bar{C}^{1}\left(\Omega_{0}\right)$. Тогда выполняется соотношение

$$
\int_{\Omega_{0}} v \Delta_{q} u d \mu=-\int_{\Omega_{0}} q \nabla v \nabla u d \mu-\int_{\partial \Omega_{0}} v(q \nabla u)_{\vec{\nu}} d \mu
$$

Из теоремы 2 немедленно следует аналог второй формулы Грина.

Теорема 3. Если $u, v \in \bar{C}^{2}\left(\Omega_{0}\right)$, то выполняется соотношение

$$
\int_{\Omega_{0}}\left(v L_{q} u-u L_{q} v\right) d \mu=\int_{\partial \Omega_{0}}\left(u(q \nabla v)_{\vec{\nu}}-v(q \nabla u)_{\vec{\nu}}\right) d \mu
$$

Теоремы 1 и 2 вместе с доказательствами можно найти, например, в [9]. В следующем пункте нам понадобится известньй, по-видимому, факт (см. следующую лемму), однако нам не удалось найти его в литературе. В силу этого мы приводим его с доказательстBOM. 
ЛЕмма 1. Пусть $G \in \mathbb{R}^{k}$ - область с кусочно-гладкой границей $\partial G$, ограниченная поверхностями $y=\psi(x), y=\varphi(x)\left(x \in \mathbb{R}^{k-1}, y \in \mathbb{R}^{1}\right)$, где $\psi(x) \geqslant \varphi(x)$. Тогда существует полохительная константа $\bar{C}$ такая, что

$$
\int_{G} u^{2} d V \leqslant \bar{C}\left(\int_{\partial G} u^{2} d S+\int_{G}(\nabla u)^{2} d V\right)
$$

где $d V$ - әлемент обгема, а $d S$ - әлемент площади гранищь $G$.

ДокАЗАтЕльство. По формуле Ньютона-Лейбница для $(x, y) \in G$ имеем

$$
u(x, y)=u(x, \varphi(x))+\int_{\varphi(x)}^{y} \frac{\partial u}{\partial y}(x, l) d l
$$

Возводя обе части этого равенства в квадрат и используя неравенство Шварца, получаем

$$
u^{2}(x, y) \leqslant 2 u^{2}(x, \varphi(x))+2 \int_{\varphi(x)}^{\psi(x)}\left(\frac{\partial u}{\partial y}\right)^{2}(x, l) d l
$$

Проинтегрируем теперь получившееся неравенство по $y$ в пределах от $\varphi(x)$ до $\psi(x)$ :

$$
\begin{aligned}
\int_{\varphi(x)}^{\psi(x)} u^{2}(x, y) d y & \leqslant 2(\psi(x)-\varphi(x)) u^{2}(x, \varphi(x))+2(\psi(x)-\varphi(x)) \int_{\varphi(x)}^{\psi(x)}\left(\frac{\partial u}{\partial y}\right)^{2}(x, l) d l \\
& \leqslant 2 d \cdot u^{2}(x, \varphi(x))+d \cdot \int_{\varphi(x)}^{\psi(x)}\left(\frac{\partial u}{\partial y}\right)^{2}(x, l) d l
\end{aligned}
$$

где $d$ - диаметр области $G$. Затем, интегрируя последовательно по $x^{i}$ в пределах от $a_{i}$ до $b_{i}$, получаем

$$
\begin{aligned}
& \int_{G} u^{2}(x, y) d y d x \\
& \quad \leqslant 2 d\left(\int_{a_{1}}^{b_{1}} \cdots \int_{a_{k-1}}^{b_{k-1}} u^{2}(x, \varphi(x)) d x^{1} \cdots d x^{k-1}+d \int_{G}\left(\frac{\partial u}{\partial y}\right)^{2}(x, l) d l d x\right) .
\end{aligned}
$$

Пользуясь далее тем, что

$$
d x=d x^{1} \cdots d x^{k-1} \leqslant \sqrt{1+\left(\frac{\partial u}{\partial x^{1}}\right)^{2}+\cdots+\left(\frac{\partial u}{\partial x^{k-1}}\right)^{2}} d x^{1} \cdots d x^{k-1}=d S
$$

заключаем, что

$$
\int_{G} u^{2} d V \leqslant 2 d \max \{1, d\}\left(\int_{\partial G} u^{2} d S+\int_{G}(\nabla u)^{2} d V\right)
$$

Лемма доказана. 
ЗАмЕчАниЕ 1. Эта лемма верна для любых односвязных областей, не обязательно выпуклых, поскольку всегда можно “диффеоморфировать” последние в вьпуклые (от диффеоморфизма будет зависеть лишь константа $\bar{C}$ в (8)). Кроме того, в силу аддитивности неравенства по области она верна и для конечносвязных областей. Нужные изменения в доказательстве здесь очевидны.

Положим $\Gamma=\bigcup_{i} \bar{\sigma}_{1 i}, \partial \Gamma=\left\{\sigma_{0 k} \in \partial \Omega_{0} ; \sigma_{0 k} \prec \sigma_{1 i} \in \Gamma\right\}$. Следующая теорема дает неравенство Пуанкаре (через $\stackrel{\circ}{C}^{1}\left(\Omega_{0}\right)$ обозначены функции, определенные на $\Omega$, обращающиеся в нуль на $\partial \Omega_{0}$, сужения которых на $\Omega_{0}$ принадлежат $\left.C^{1}\left(\Omega_{0}\right)\right)$.

ТЕорема 4. Пусть $u \in \stackrel{\circ}{C}^{1}\left(\Omega_{0}\right)$. Тогда существует константа $\widehat{C}$ такая, что ${ }^{1}$

$$
\int_{\Gamma} q u^{2} d \mu \leqslant \widehat{C} \int_{\Gamma} p u^{\prime 2} d \mu .
$$

ДокАЗАТЕЛЬСТво. Рассмотрим путь в $\Gamma$, соединяющий $x \in \Gamma$ с $a \in \partial \Gamma$, и ориентируем его от $a$ к $x$. Пусть $a_{1}, a_{2}, \ldots, a_{k}$ - последовательность 0-мерных стратов, проходимых вдоль пути от $a \mathrm{k} x$. Тогда

$$
u(x)=\int_{a_{1}}^{a_{2}} u^{\prime} d l+\cdots+\int_{a_{k-1}}^{a_{k}} u^{\prime} d l+\int_{a_{k}}^{x} u^{\prime} d l
$$

здесь мы воспользовались непрерьвностью $u$ и тем, что $u\left(a_{k}\right)=0$. Из неравенства Шварца для сумм получаем

$$
u^{2}(x) \leqslant k\left(\left(\int_{a_{1}}^{a_{2}} u^{\prime} d l\right)^{2}+\cdots+\left(\int_{a_{k}}^{x} u^{\prime} d l\right)^{2}\right) .
$$

Обозначая через $l_{0}$ максимальную из длин 1 -мерных стратов в Г и используя неравенство Шварца для интегралов, получим (с очевидными огрублениями)

$$
u^{2}(x) \leqslant k l_{0}\left(\int_{a_{1}}^{a_{2}} u^{\prime 2} d l+\cdots+\int_{a_{k}}^{a_{k+1}} u^{\prime 2} d l\right)
$$

где $a_{k+1}$ является еще одним концом (помимо $a_{k}$ ) 1-мерного страта, содержащего $x$. Отсюда следует оценка

$$
u^{2}(x) \leqslant M l_{0} \int_{\Gamma} u^{\prime 2} d \mu,
$$

где $M$ - число 0-мерных стратов в Г. Правая часть неравенства (9) не зависит от $x$, поэтому, в частности,

$$
u^{2}(b) \leqslant M l_{0} \int_{\Gamma} u^{\prime 2} d \mu
$$

для любого 0-мерного страта $\{b\}$ из Г. Интегрируя (9) по каждому 1-мерному страту и суммируя, получим

$$
\sum \int_{\sigma_{1 i}} u^{2}(x) d l \leqslant N M l_{0} \int_{\Gamma} u^{\prime 2} d \mu
$$

\footnotetext{
${ }^{1}$ Напомним еще раз, что 0-мерный градиент мы считаем нулевым, так что вклад в правую часть неравенства от 0 -мерных стратов нулевой.
} 
где через $N$ обозначается число одномерных стратов в $Г$. Добавляя к обеим частям последнего выражения неравенство (10), выводим

$$
\int_{\Gamma} u^{2} d \mu=\sum \int_{\sigma_{1 i}} u^{2}(x) d l+\sum u^{2}(b) \leqslant(N+M) M l_{0} \int_{\Gamma}{u^{\prime 2}}^{2} d \mu=\widehat{C} \int_{\Gamma} u^{\prime 2} d \mu
$$

Теорема доказана.

Конечно, огрубления, проведенные в ходе доказательства, слишком сильны и константа в итоговом неравенстве далека от оптимальной. Но для рассмотрения наших вопросов это не имеет значения. Кроме того, в качественном отношении приведенное неравенство “сильнее” неравенства Пуанкаре. В самом деле, в случае, когда Г состоит из двух отрезков $[a, \xi],[\xi, b]$, наше неравенство дает

$$
\int_{\Gamma} u^{2} d \mu=\int_{a}^{b} u^{2} d l+u^{2}(\xi) \leqslant \widehat{C} \int_{a}^{b} u^{\prime 2} d l
$$

в то время как обычное неравенство Пуанкаре вьглядит так:

$$
\int_{a}^{b} u^{2} d l \leqslant \widehat{C} \int_{a}^{b} u^{\prime 2} d l
$$

4. Задача Дирихле. Нас будет интересовать краевая задача

$$
L_{q} u=f,\left.\quad u\right|_{\partial \Omega_{0}}=0
$$

с квадратично-суммируемьми на замькании каждого страта коэффициентами $p, q$ и правой частью $f$. При этом $q$ предполагается неотрицательной.

Слабым решением задачи (11), как обычно, будем называть функции из пространства $\stackrel{\circ}{H}^{1}\left(\Omega_{0}\right)$ (см. ниже), удовлетворяющие для любой $\varphi \in \stackrel{\circ}{H}^{1}\left(\Omega_{0}\right)$ тождеству

$$
-\int_{\Omega_{0}}(p \nabla u \nabla \varphi+q u \varphi) d \mu=\int_{\Omega_{0}} f \varphi d \mu
$$

Разумеется, под $\nabla u$ понимается слабьй градиент.

Пространство $\stackrel{\circ}{H}^{1}\left(\Omega_{0}\right)$ получается пополнением $\stackrel{\circ}{C}^{1}\left(\Omega_{0}\right)$ по норме

$$
\|u\|=\sqrt{\langle u, u\rangle}
$$

порожденной скалярным произведением

$$
\langle u, v\rangle=\int_{\Omega_{0}}(p \nabla u \nabla v+q u v) d \mu .
$$

Расмотрим аддитивный, однородный функционал

$$
\Phi_{f}(\phi)=\int_{\Omega_{0}} f \phi d \mu, \quad \phi \in \stackrel{\circ}{C}^{1}\left(\Omega_{0}\right) .
$$


ЛЕмма 2. Функиионал $\Phi_{f}$ непрерывен на $\stackrel{\circ}{C}^{1}\left(\Omega_{0}\right)$ по норме $(13)$.

Для доказательства этой леммы нам потребуется аналог неравенства Пуанкаре, а именно

Лемма 3. Для любой функиии $и \in \stackrel{\circ}{C}^{1}\left(\Omega_{0}\right)$ выполняется соотношение

$$
\int_{\Omega_{0}} u^{2}(x) d \mu \leqslant C \int_{\Omega_{0}} p(\nabla u)^{2}(x) d \mu
$$

с не зависящей от и константой $C$.

ДокАЗАТЕЛЬСТво. В силу замечания к лемме 1 имеем (применяя его к каждому страту)

$$
\int_{\sigma_{k j}} u^{2} d \mu \leqslant C_{k j}\left(\sum_{\sigma_{k-1, j} \prec \sigma_{k j}} \int_{\sigma_{k-1, i}} u^{2} d \mu+\int_{\sigma_{k j}}(\nabla u)^{2} d \mu\right) .
$$

Поскольку $\left.u\right|_{\partial \Omega_{0}}=0$, интегралы по $(k-1)$-мерньм стратам, входящим в $\partial \Omega_{0}$, обращаются в нуль. К остальным же $(k-1)$-мерньм стратам вновь применим упомянутое замечание. Для каждого из них получим неравенство типа (14) со своими константами. Обозначая через $C_{k j}^{1}$ наибольшую из них и подставляя эти неравенства в правую часть (14), будем иметь

$$
\int_{\sigma_{k j}} u^{2} d \mu \leqslant C_{k j}\left(C_{k j}^{1} \sum \sum \int_{\sigma_{k-2, j_{2}}} u^{2} d \mu+C_{k j}^{1} \sum \int_{\sigma_{k-1, j_{1}}}(\nabla u)^{2} d \mu+\int_{\sigma_{k j}}(\nabla u)^{2} d \mu\right) .
$$

Продолжая этот процесс индуктивно вплоть до 1-мерньх стратов, получим в итоге неравенство вида

$$
\begin{aligned}
\int_{\sigma_{k j}} u^{2} d \mu \leqslant & C_{k j}\left(C_{k j}^{k-1} \cdots C_{k j}^{1} \sum \cdots \sum \int_{\sigma_{1 j_{k-1}}} u^{2} d \mu\right. \\
& +C_{k j}^{k-2} \cdots C_{k j}^{1} \sum \cdots \sum \int_{\sigma_{2 j_{k-2}}}(\nabla u)^{2} d \mu+\cdots \\
& \left.+C_{k j}^{1} \sum \int_{\sigma_{k-1, j_{1}}}(\nabla u)^{2} d \mu+\int_{\sigma_{k j}}(\nabla u)^{2} d \mu\right)
\end{aligned}
$$

В правой части (15) фигурируют интегралы от $(\nabla u)^{2}$ по всем стратам $\sigma_{m i}(m \geqslant 2)$, входящим в $\bar{\sigma}_{k j}$, кроме граничных, и интегралы от $u^{2}$ по одномерным стратам (одномерному “остову” $\sigma_{k j}$ ), вновь не включенным в $\partial \Omega_{0}$. Неравенство (15) очевидньг образом огрубляется до следующего:

$$
\int_{\sigma_{k j}} u^{2} d \mu \leqslant \bar{C}_{k j}\left(\int_{\Gamma} u^{2} d \mu+\int_{\Omega_{0}}(\nabla u)^{2} d \mu\right)
$$

где

$$
\bar{C}_{k j}=C_{k j} \cdot \max \left(\prod_{i=1}^{k-1} C_{k j}^{i}, \prod_{i=1}^{k-2} C_{k j}^{i}, \ldots, C_{k j}^{1}\right)
$$


Последнее в силу теоремы 4 оценивается выражением

$$
\bar{C}_{k j}\left(\widehat{C} \int_{\Gamma} u^{2} d \mu+\int_{\Omega_{0}}(\nabla u)^{2} d \mu\right) \leqslant \widetilde{C}_{k j} \int_{\Omega_{0}}(\nabla u)^{2} d \mu .
$$

Суммируя по всем стратам из $\Omega_{0}$, заключаем, что

$$
\int_{\Omega_{0}} u^{2} d \mu \leqslant C \int_{\Omega_{0}}(\nabla u)^{2} d \mu .
$$

Поскольку

$$
\int_{\Omega_{0}} p(\nabla u)^{2} d \mu \geqslant \alpha \int_{\Omega_{0}}(\nabla u)^{2} d \mu
$$

окончательно имеем

$$
\int_{\Omega_{0}} u^{2} d \mu \leqslant \widetilde{C} \int_{\Omega_{0}} p(\nabla u)^{2} d \mu
$$

где константа $\widetilde{C}=C / \alpha$ не зависит от функции $u$.

ДокАЗАТЕЛЬСтво ЛЕммы 2. В силу неравенства Шварца

$$
\begin{aligned}
\left|\Phi_{f}(\phi)\right| & \leqslant\left(\int_{\Omega_{0}} f^{2} d \mu\right)^{1 / 2}\left(\int_{\Omega_{0}} \phi^{2} d \mu\right)^{1 / 2} \leqslant C\left(\int_{\Omega_{0}} f^{2} d \mu\right)^{1 / 2}\left(\int_{\Omega_{0}} p(\nabla \phi)^{2} d \mu\right)^{1 / 2} \\
& \leqslant C\left(\int_{\Omega_{0}} f^{2} d \mu\right)^{1 / 2}\left(\int_{\Omega_{0}}\left[p(\nabla \phi)^{2} d \mu+q \phi^{2}\right]\right)^{1 / 2}=\widetilde{C}\|\phi\|_{H^{1}\left(\Omega_{0}\right)} .
\end{aligned}
$$

Второе от начала неравенство обеспечивается леммой 3 .

Таким образом, мы доказали ограниченность функционала $\Phi_{f}$ по норме $\stackrel{\circ}{H}^{1}\left(\Omega_{0}\right)$ на множестве функций из $\stackrel{\circ}{C}^{1}\left(\Omega_{0}\right)$, а следовательно, и непрерьвность его по этой норме.

Лемма доказана.

Поскольку $\stackrel{\circ}{C}^{1}\left(\Omega_{0}\right)$ плотно в $\stackrel{\circ}{H}^{1}\left(\Omega_{0}\right)$, функционал $\Phi_{f}$ в силу доказанной леммы допускает продолжение до непрерьвного функционала $\widetilde{\Phi}_{f}$ в $\stackrel{\circ}{H}^{1}\left(\Omega_{0}\right)$, которьй по теореме о представлении функционала в гильбертовом пространстве при некотором однозначно определяемом $u \in \stackrel{\circ}{H}^{1}\left(\Omega_{0}\right)$ имеет вид

$$
\widetilde{\Phi}_{f}(\psi)=\langle u, \psi\rangle, \quad \psi \in \stackrel{\circ}{H}^{1}\left(\Omega_{0}\right)
$$

С другой стороны, имеет место представление

$$
\widetilde{\Phi}_{f}=\int_{\Omega_{0}} f \psi d \mu
$$

Из (16), (17) получаем

$$
\langle u, \psi\rangle=\int_{\Omega_{0}} f \psi d \mu,
$$

что эквивалентно равенству (12). Таким образом, имеет место 
ТЕорема 5. При перечисленных выше условиях на $p, q, f$ задача (11) имеет единственное слабое решение $u \in \stackrel{\circ}{H}^{1}\left(\Omega_{0}\right)$.

Заметим, что из определения $\stackrel{\circ}{H}^{1}\left(\Omega_{0}\right)$ следует существование последовательности $\left\{u_{j}\right\}$ функций из $\stackrel{C}{C}^{1}\left(\Omega_{0}\right)$ такой, что при $j \rightarrow \infty u_{j} \rightarrow u$ в $\stackrel{\circ}{H}^{1}\left(\Omega_{0}\right)$. Тогда для любой $\psi \in \stackrel{C}{C}^{2}\left(\Omega_{0}\right)$ имеем в силу первой и второй формул Грина

$$
\begin{aligned}
\int_{\Omega_{0}} f \psi d \mu & =\langle u, \psi\rangle=\lim _{j \rightarrow \infty}\left\langle u^{j}, \psi\right\rangle=\lim _{j \rightarrow \infty} \int_{\Omega_{0}}\left(p \nabla u^{j} \nabla \psi+q u^{j} \psi\right) d \mu \\
& =\lim _{j \rightarrow \infty} \int_{\Omega_{0}} \psi L_{q} u^{j} d \mu=\lim _{j \rightarrow \infty} \int_{\Omega_{0}} u^{j} L_{q} \psi d \mu=\int_{\Omega_{0}} u L_{q} \psi d \mu
\end{aligned}
$$

тем самым, если $p$ слабо дифференцируемо, то $u$ является и обобщенньг решением (11).

5. Задача Дирихле для эллиптического оператора четвертого порядка. Как видим, использование дивергенции по мере позволяет в некоторой степени следовать классическому образцу при обсуждении разрешимости задачи Дирихле для уравнения второго порядка. Естественность такого подхода подтверждается еще и тем, что итерация описьваемого дифференциального оператора, которую было бы разумным назвать бигармоническим оператором на стратифицированных множествах, возникает (см. [12]) при описании деформации систем, составленных из стержней и пластин. Рассмотрим на $\bar{C}^{4}\left(\Omega_{0}\right)$ дифференциальньй оператор

$$
\mathscr{L} u=\left(\Delta_{p}\left(r \Delta_{p}\right)-\Delta_{q}+k\right) u,
$$

$k(x) \geqslant 0$ - локально интегрируемая на $\Omega_{0}$ функция, $r \in \bar{C}_{\sigma}^{2}\left(\Omega_{0}\right), q \in C_{\sigma}^{1}\left(\Omega_{0}\right)$ строго положительны. Рассмотрим также задачу

$$
\begin{aligned}
& \mathscr{L} u(x)=f(x), \quad x \in \Omega_{0}, \\
& u(x)=\frac{\partial u(x)}{\partial y^{\alpha}}=0, \quad x \in \partial \Omega_{0},
\end{aligned}
$$

где $f(x)$ - локально интегрируемая на $\Omega_{0}$ функция.

Краевой задачей вида (18) описываются, в частности, малые деформации натянутой системы, состоящей из шарнирно сочлененных между собой пластинок и стержней, помешенной на упругое основание. Требование положительности $q$ было введено во избежание эффекта "прощелкивания".

Слабое решение (18) ищется по той же схеме, что и для оператора второго порядка. При этом скалярное произведение имеет вид

$$
\langle u, v\rangle=\int_{\Omega_{0}}\left(\Delta_{p} u r \Delta_{p} v+q \nabla u \nabla v+k u v\right) d \mu,
$$

а формулы Грина немного более громоздки.

Теорема 6. Пусть $w \in \bar{C}_{\sigma}^{4}\left(\Omega_{0}\right), v \in \bar{C}^{2}\left(\Omega_{0}\right)$. Тогда имеет место соотношение

$$
\begin{gathered}
\int_{\Omega_{0}} v \mathscr{L} w d \mu=\int_{\Omega_{0}}\left(k v w+q \nabla v \nabla w+r \Delta_{p} v \Delta_{p} w\right) d \mu+\int_{\partial \Omega_{0}} v(q \nabla w)_{\vec{\nu}} d \mu \\
-\int_{\partial \Omega_{0}}\left[v\left(p \nabla\left(r \Delta_{p} w\right)\right)_{\vec{\nu}}-r \Delta_{p} w(p \nabla v)_{\vec{\nu}}\right] d \mu
\end{gathered}
$$


TEOpema 7. Ecлu $u, v \in \bar{C}^{4}\left(\Omega_{0}\right)$, mo

$$
\begin{aligned}
\int_{\Omega_{0}}(v \mathscr{L} w-w \mathscr{L} v) d \mu=\int_{\partial \Omega_{0}} & {\left[v(q \nabla w)_{\vec{\nu}}-v\left(p \nabla\left(r \Delta_{p} w\right)\right)_{\vec{\nu}}+r \Delta_{p} w(p \nabla v)_{\vec{\nu}}\right] d \mu } \\
& -\int_{\partial \Omega_{0}}\left[w(q \nabla v)_{\vec{\nu}}-w\left(p \nabla\left(r \Delta_{p} v\right)\right)_{\vec{\nu}}+r \Delta_{p} v(p \nabla w)_{\vec{\nu}}\right] d \mu .
\end{aligned}
$$

Аналог неравенства Пуанкаре

$$
\int_{\Omega_{0}} u^{2}(x) d \mu \leqslant C \int_{\Omega_{0}}\left(\Delta_{p} u\right)^{2}(x) d \mu,
$$

получаемьй в данном случае итерацией уже доказанного неравенства, позволяет применить теорему о представлении в пространстве $\stackrel{\circ}{H}^{2}\left(\Omega_{0}\right)$, вводящемся пополнением $\stackrel{\circ}{C}^{2}\left(\Omega_{0}\right)$ по норме, порожденной скалярным произведением (19).

\section{СПИСОК ЦИТИРОВАННОЙ ЛИТЕРАТУРЫ}

[1] Nicaise S. Estimeés du spectre du laplacian sur un réseau topologique fini // C. R. Acad. Sci. 1986. V. 303. P. 343-346.

[2] Павлов Б.С., Фаддеев М.Д. Модель свободных электронов и задача рассеяния // ТМФ. 1983. Т. 55. № 2. С. 257-269.

[3] Пенкин О. М., Покорный Ю. В., Провоторова Е. Н. Об одной векторной краевой задаче // Сб. "Краевые задачи". Пермь: Изд-во ППИ, 1983. С. 64-70.

[4] Ali Mehmeti F. Nonlinear Waves in Networks. Berlin: Academie-Verlag, 1994.

[5] Копачевский Н. Д., Крейн С. Г., Нго Зуй Кан. Операторные методы в линейной гидродинамике. М.: Наука, 1989.

[6] Жиков В.В. Связность и усреднение. Примеры фрактальной проводимости // Матем. сб. 1996. T. 187. № 8. С. 3-40.

[7] Покорный Ю.В., Пенкин О.М.О теоремах сравнения для уравнений на графах // Дифференц. уравнения. 1989. Т. 25. № 7. С. 1141-1150.

[8] Пенкин О. М. О принципе максимума для эллиптического уравнения на двумерном клеточном комплексе // Докл. РАН. 1997. Т. 352. №4. С. 462-465.

[9] Пенкин О.М., Покорный Ю. В. О дифференциальных неравенствах для эллиптических уравнений на сложных многообразиях // Докл. РАН. 1998. Т. 360. № 4. С. 456-458.

[10] Пенкин О.М., Покорный Ю. В. О несовместных неравенствах для эллиптических уравнений на стратифицированных множествах // Дифференц. уравнения. 1998. Т. 34. № 8 . C. $1107-1113$.

[11] Пенкин О.М., Богатов Е. М., Кашкаров Ю.М. О разрешимости эллиптических краевых задач на стратифицированных множествах // Дифференц. уравнения. 1998. Т. 34. № 9. C. $1289-1290$.

[12] Богатов Е. М. О разрешимости одной задачи линейной теории упругости // Сб. статей аспирантов и студентов математического факульета ВГУ. Воронеж: Изд-во ВГУ, 1999. C. 9-15.

Воронежский государственный университет 